\title{
Metabolism of Platelet-activating Factor in Isolated Perfused Rat Lung
}

\author{
P. E. Haroldsen," N. F. Voelkel, ${ }^{\ddagger}$ J. E. Henson, ${ }^{5}$ P. M. Henson, ${ }^{5}$ and R. C. Murphy* \\ *Department of Pharmacology, and ${ }^{\ddagger}$ Cardiovascular Pulmonary Research Laboratory and Webb-Waring Lung Institute, University of \\ Colorado Health Sciences Center; and ${ }^{\S}$ National Jewish Center for Immunology and Respiratory Medicine, Denver, Colorado 80206
}

\begin{abstract}
The administration of platelet-activating factor (PAF) into the airway system of the lung is known to cause profound effects, yet little is known about the metabolism of this active lipid mediator. ${ }^{3} \mathrm{H}$-Labeled PAF administered into the airway of isolated rat lungs was rapidly and extensively metabolized. The tissue retained $96 \%$ of the administered radiolabel while the perfusate contained $4 \%$. Characterization of the tissue retained lipid indicated metabolism into lyso-PAF (3.3\%), phosphatidylcholine (82.3\%), neutral lipid (1.7\%) and intact PAF (10.2\%). Analysis of tissue phosphatidylcholine by mass spectrometric techniques revealed metabolism of PAF to $\mathbf{i}-0$-hexadecyl-2-arachidonoylGPC, which represented 20-23\% of the administered radiolabeled hexadecyl-PAF. These findings support the hypothesis that a relationship between PAF and arachidonate metabolism exists at the intact organ level. Autoradiographic analysis of the cellular distribution of the radiolabeled PAF metabolites in the lung tissue indicated labeling of two cell types, the alveolar type II cell and the nonciliated bronchiolar epithelial cell (Clara cell).
\end{abstract}

\section{Introduction}

Platelet-activating factor (PAF), ${ }^{1}$ identified as 1 - $O$-alkyl-2-acetyl$s n$-glycero-3-phosphocholine (1), is a unique phospholipid possessing many potent biologic activities that have led to its implication as an important mediator of inflammatory reactions (2). The lung is known as a site of synthesis for lipid mediators such as the leukotrienes, prostaglandins, thromboxanes, and PAF. The icosanoids are pharmacologically active in the lung, and cause bronchoconstriction (3), vasoconstriction (4), and vasopermeability (5). PAF also has profound effects on the lung (6), similar to the peptidoleukotrienes, which include vasoconstriction (7), bronchoconstriction (8), edema formation $(7,9)$, and the recently described pulmonary vasodilation when low PAF concentrations were tested (10). Although the pulmonary metabolism of the icosanoids (11) has been studied in some detail, little is known about the metabolism of PAF by this organ.

Address correspondence to Prof. Murphy, Department of Pharmacology C236, U. of Colorado Health Sciences Center, Denver, CO 80262.

Received for publication 23 October 1986 and in revised form 28 January 1987.

1. Abbreviations used in this paper: DPPC, dipalmitoylglycerophosphocholine; GC/MS, gas chromatography/mass spectrometry; GPC, glycerophosphocholine; NL, neutral lipid; PAF, platelet-activating factor, PFB, pentafluorobenzoate, TLC, thin-layer chromatography.

J. Clin. Invest.

(c) The American Society for Clinical Investigation, Inc.

$0021-9738 / 87 / 06 / 1860 / 08 \$ 1.00$

Volume 79, June 1987, 1860-1867
The distribution of radiolabeled PAF injected into a rat has been reported by Blank et al. (12) as well as the partial characterization of its metabolites. At the cellular level, PAF has been well documented (13-18) to be rapidly inactivated by deacetylation followed by reacylation with long chain fatty acyl groups. In some cell types, a high degree of reacylation with arachidonic acid has been reported (13). Cells forming this unique 1-O-alkyl-2-arachidonoyl glycerophosphocholine (GPC) from PAF include the human neutrophil $(13,14)$, rat alveolar macrophage $(15)$, human umbilical vein endothelial cell (16), rat capillary endothelial cells (17), and human platelet (18). In view of the actions of PAF in the lung shared by arachidonic acid metabolites and of the biochemical connection between PAF and arachidonate at the cellular level, it appeared important to determine whether such a metabolic link would exist at the whole organ level. We wished to investigate whether the metabolism of PAF in the whole lung was similar to that detected on the cellular level. We also wondered whether PAF uptake was an ubiquitous phenomenon or whether there were privileged lung cell types involved in the processing of this bioactive material. The latter question is of importance in view of recent reports suggesting a role of PAF in human lung maturation (19) as well as the potential contribution of this molecule to airway inflammatory processes. In this study, the metabolic profile of PAF metabolism and cellular involvement was determined in the isolated perfused rat lung. The intratracheal route of administration was chosen in order to mimic the appearance of this compound in the airways, to determine whether PAF applied via this route caused edema or vasoconstriction, and to determine the metabolic fate in the airspace of this potential mediator of lung inflammation or lung injury.

\section{Methods}

Labeled PAF (1-L-[ $\left[{ }^{3} \mathrm{H}\right]$ alkyl-2-acetyl-glycerol-3-phosphocholine, 45-59.5 $\mathrm{Ci} / \mathrm{mmol}$ ), lyso-PAF (1-O-[ $\left.{ }^{3} \mathrm{H}\right]$ alkyl-2-lyso-sn-glycerol-3-phosphocholine, $45 \mathrm{Ci} / \mathrm{mmol}$ ), and dipalmitoylglycerophosphocholine (DPPC [1,2-dihexadecanoyl-sn-glycerol-3-phospho[methyl $\left.{ }^{3} \mathrm{H}\right]$ choline, $\left.\left.5 \mathrm{Ci} / \mathrm{mmol}\right]\right)$ were obtained from New England Nuclear (Boston, MA). The [ $\left.{ }^{3} \mathrm{H}\right] \mathrm{PAF}$ used for initial experiments had a composition of 52\% hexadecyl PAF, $18 \%$ octadecyl PAF, 9\% tetradecyl PAF, and 21\% uncharacterized alkyl PAF. Subsequent experiments were carried out with $\left[{ }^{3} \mathrm{H}\right] \mathrm{PAF}$, which was $99 \%$ hexadecyl PAF. Both $\left[{ }^{3} \mathrm{H}\right] \mathrm{PAF}$ and $\left[{ }^{3} \mathrm{H}\right]$ lyso-PAF were purified by a silicic acid column chromatography before use to remove labeled neutral lipid (NL) contaminants. Unlabeled PAF, lyso-PAF, GPC and glycerophosphoethanolamine, essentially fatty acid free bovine serum albumin ( $<0.005 \%$ fatty acid, fraction 5$)$, trizma base and phospholipase C (bacillus cereus type XIII) were obtained from Sigma Chemical Co. (St. Louis, MO).

Open column chromatography was carried out with silica gel (Silicar CC4, 100 mesh; Mallinckrodt Inc., Science Products Div., St. Louis, MO). Ultrasphere ODS $(5 \mu \mathrm{m}, 0.45 \times 25 \mathrm{~cm})$ was used for reverse-phase high performance liquid chromatography (HPLC) with a 5- $\mu \mathrm{m}$ ODS, 5mm precolumn cartridge (Waters Assoc., Millipore Corp., Milford, MA). 
Thin-layer chromatography (TLC) was carried out on $250-\mu \mathrm{m}$ silica gel, GHL plates from Analtech Inc. (Newark, DE). HPLC and TLC solvents were obtained from Fisher Scientific Co. (Pittsburgh, PA). Derivatization reagents (3,5-dinitrobenzoylchloride, pentafluorobenzoyl chloride, and 2,2-dimethoxypropane) were obtained from Aldrich Chemical Co. (Milwaukee, WI) and were used without further purification. The trideuteroPAF internal standard was synthesized as previously described (20). Phospholipid molecular species standards were synthesized by the addition of $100 \mu \mathrm{l}$ chloroform (ethanol free) and $75 \mathrm{mg}$ each of the corresponding free fatty acid and fatty acyl chloride (Nu-Chek Prep, Inc., Elysian, MN) to $1 \mathrm{mg}$ lyso-PAF. After stirring for $24 \mathrm{~h}$ at room temperature, the resulting GPC was purified by TLC. The chemical characterization and analysis of the purified, synthetic GPC was carried out by fast atom bombardment mass spectrometry (21).

Lung preparation and instillation of $P A F$. Lungs from anesthetized male rats $(300-400 \mathrm{~g})$ were isolated, ventilated, and perfused as previously described $(11,12)$, with the exception that the perfusion medium contained 3\% albumin in order to trap PAF or its metabolites appearing in the lung effluent. After discarding the first $20 \mathrm{ml}$ of perfusate, the lung was equilibrated for $30 \mathrm{~min}$ with recirculation of the perfusion medium. After this period, perfusion and ventilation were interrupted for $\sim 30 \mathrm{~s}$ to allow the instillation of the lipid substrate into the airway. The lipids to be instilled were suspended in $0.07 \mathrm{ml}$ saline containing $0.5 \%$ bovine serum albumin (wt/vol). PAF (150 ng, $7 \mu \mathrm{Ci}$ ), lyso-PAF (150 ng, $7 \mu \mathrm{Ci}$ ), and DPPC ( $150 \mathrm{ng}, 10 \mu \mathrm{Ci}$ ) were administered by a microliter syringe connected to a flexible polyethylene catheter $(0.001$ in.) that was advanced through the trachea into the peripheral region of the lower right lobe and injected as a bolus. After instillation, the lung was ventilated and perfused in a nonrecirculating manner for an additional $15 \mathrm{~min}$, during which time 0.5 -min effluent fractions were collected and saved for further analysis. The syringe and catheter were flushed with chloroform/methanol (1:1) to collect uninjected radiolabeled lipid. This mode of bolus instillation of PAF was not intended to assess in a quantitative manner the relative distribution and uptake of PAF either by larger or by more peripheral airways. Perfusion flow at constant pressure was monitored throughout the experiment and was not altered by instillation.

Lipid extraction of lung tissue and effluent. After the perfusion period, the lung was immersed into a beaker containing cold chloroform $/ \mathrm{meth}$ anol $\left(-30^{\circ} \mathrm{C}, 1: 2\right)$. Subsequently, the lung was thawed to allow mincing and homogenization (Polytron; Brinkman Instruments Inc., Westbury, NY). The homogenate was extracted twice by the method of Bligh and Dyer (23). The lung effluent was brought to $80 \%$ ethanol to precipitate albumin and additional proteins and then centrifuged at 1,500 $\mathrm{g}$. The ethanol supernatant was taken to dryness in vacuo and the residue resuspended in methanol/water (1:1) followed by chloroform/methanol (1:1) and subjected to phase separation by the method of Bligh and Dyer (23). The radiolabeled lipids in a lower phase were separated by the polar, then nonpolar TLC systems described below. The yield of $\left[{ }^{3} \mathrm{H}\right] \mathrm{PAF}$ recovered from the lung tissue was expressed as the tissue-extractable radioactivity divided by the total administered dose. Since PAF can absorb to surfaces, the administered dose was the difference between the tritium content in the instillation solution and that which was not injected but recovered from syringe and tubing.

Derivatization procedures. Lyso-PAF was converted to the 1-O-alkyl 2,3-isopropylidine derivative as previously described (24). PAF and GPC metabolites were converted to diglycerides by treatment with phospholipase C. Briefly, $1 \mathrm{ml}$ of $0.1 \mathrm{M}$ Tris-HCl buffer ( $\mathrm{pH} 7.5$ ), $40 \mathrm{U}$ of enzyme, and $2 \mathrm{ml}$ of diethyl ether were added to the dried lipid substrate. The two-phase mixture was shaken at room temperature for $2 \mathrm{~h}$, after which time the ether layer was removed; this was followed by extraction of the aqueous layer a second time with $2 \mathrm{ml}$ ether. After removal of the organic solvent under a stream of nitrogen and lyophilization, the diglycerides were derivatized for analysis by negative ion electron capture mass spectrometry. The pentafluorobenzoyl derivative of PAF was formed by dissolving the 1-O-alkyl, 2-acetyl diglyceride in $0.05 \mathrm{ml}$ of hexane and 0.2 $\mathrm{ml}$ pentafluorobenzoyl chloride followed by heating at $100^{\circ} \mathrm{C}$ for $1.5 \mathrm{~h}$ (25). The dinitrobenzoyl derivatives of the GPC molecular species diglycerides were formed by the method of Kito et al. (26). Briefly, the diglyceride was dissolved in $0.4 \mathrm{ml}$ pyridine (freshly distilled) and $25 \mathrm{mg}$ of 3,5-dinitrobenzoyl chloride was added. The reaction was allowed to continue for $1 \mathrm{~h}$ at $65^{\circ} \mathrm{C}$. Water $(0.5 \mathrm{ml})$ was added, followed by $2.0 \mathrm{ml}$ of $0.1 \mathrm{mM}$ Tris- $\mathrm{HCl}$ buffer (pH 7.5) 5 min later. Hexane (three times, $1.5 \mathrm{ml}$ ) was then added to the aqueous solution to extract the diglyceride dinitrobenzoates and the derivatized species were separated by TLC using a nonpolar solvent system.

HPLC and TLC chromatography. Lung tissue and lung effluent extracts were fractionated into their constitutive classes by TLC using the polar solvent system: chloroform/methanol/acetic acid/water (50:30:8:5, by volume). Each separated lipid was eluted from the TLC band by sequential washing of the silicic acid. For phosphatidylcholine, $1.5 \mathrm{ml} \mathrm{CHCl} / 3 / \mathrm{MeOH}(20: 80, \mathrm{vol} / \mathrm{vol})$ with $1.5 \mathrm{ml} \mathrm{H}_{2} \mathrm{O}$ was added to the scraped TLC band followed by vortexing and centrifugation. For the phosphatidylethanolamine class, the eluting solvent system was $1.5 \mathrm{ml}$ $\mathrm{CHCl}_{3} / \mathrm{MeOH}(75: 25 \mathrm{vol} / \mathrm{vol})$ with $1.5 \mathrm{ml} \mathrm{H} \mathrm{H}_{2}$; $\mathrm{NL}$ were eluted with $1.5 \mathrm{ml} \mathrm{CHCl} / \mathrm{MeOH}(90: 10 \mathrm{vol} / \mathrm{vol})$. Separation of NL was carried out using the solvent system chloroform/methanol/acetic acid (98:2.2:1) in order to identify monoglycerides, diglycerides, and triglycerides. The nonpolar solvent system benzene/hexane/diethyl ether (50:45:10) was used for the purification of diglyceride dinitrobenzoates. Essentially the same solvent system with one-half the amount of diethyl ether was used for the separation of the pentafluorobenzoate (PFB) derivatives. The dinitrobenzoate and PFB derivatives were eluted from the silicic acid with diethyl ether. Radioactive bands separated by the TLC systems were detected by a radioactive linear analyzer (TLC Tracemaster; Berthold, Wildbod, FRG).

Diglyceride dinitrobenzoates were analyzed by reverse-phase HPLC as previously described (26) except using the mobile phase acetonitrile/ isopropanol (85:15). The effluent from the HPLC was monitored by scintillation counting using an on-line radioactive monitor (Ramona D IN/US). Quantitation of total radioactivity in each radioactive HPLC peak was determined by liquid scintillation counting techniques. Radioactive labeled metabolites were tentatively identified by retention time comparison with synthetic standards.

Mass spectrometry. Mass spectral analyses were performed on a Nermag R-1010C quadrupole mass spectrometer coupled to a Varian gas chromatograph with a 5-m DB-1 (J \& W Scientific, Rancho Cordova, CA) using helium as carrier gas. The cyclic isopropylidine derivatives were analyzed by gas chromatography/mass spectrometry (GC/MS) in the positive electron impact mode $(70 \mathrm{eV})$. The 1-O-alkyl-2-acetyl pentafluorobenzoates were analyzed by GC/MS in the negative ion electron capture mode using methane as the moderating gas at 0.1 torr. The diglyceride dinitrobenzoates were analyzed by negative ion desorption chemical ionization using methane as the moderating gas. Solutions of purified dinitrobenzoates were added to the tungsten wire of the desorption probe $(5-10 \mu \mathrm{l})$ and the probe then heated in the ion source by a programmed current $0-300 \mathrm{~mA}$ at $10 \mathrm{~mA} / \mathrm{s}$.

Autoradiography. After instillation of the radiolabeled lipids PAF (100 ng, $25 \mu \mathrm{Ci})$, lyso-PAF (100 ng, $25 \mu \mathrm{Ci}$ ), and DPPC (100 ng, 25 $\mu \mathrm{Ci})$, followed by a 15 -min perfusion period, the lung tissue was fixed briefly with $1.5 \%$ glutaraldehyde in $0.1 \mathrm{M}$ cacodylate buffer (pH 7.3, 330 mosmol) by intravascular and intratracheal infusions. After $30 \mathrm{~min}$, glutaraldehyde was drained from the lung and $1.0 \%$ osmium tetroxide in $0.1 \mathrm{M}$ cacodylate buffer ( $\mathrm{pH} 7.3$ ) was instilled intratracheally. The specimen was fixed for $3 \mathrm{~h}$ at $4^{\circ} \mathrm{C}$. Areas were selected from each lobe for $1-\mu \mathrm{m}$ blocks that were further fixed in $1.0 \%$ osmium tetroxide, $0.1 \mathrm{M}$ cacodylate buffer overnight at $4^{\circ} \mathrm{C}$. After this treatment, the sample was dehydrated in graded percentages of acetone as quickly as possible. Clearing and infiltration were performed using propylene oxide and were followed by an Epon/Araldite 502 mixture. The tissue was embedded in pure Epon/Araldite 502 and cured at $60^{\circ} \mathrm{C}$. $1-\mu \mathrm{m}$ sections were cut on an LKB Nova ultramicrotome and sections were coated with Kodak NTB-2 nuclear track emulsion using standardized procedures, and stored at $4^{\circ} \mathrm{C}$ for 7 and $14 \mathrm{~d}$. The emulsion was developed using Kodak developer D-19 for 4 min followed by a distilled water wash for $10 \mathrm{~s}$, then using Kodak fixer for $5 \mathrm{~min}$ before a final distilled water wash. Sections were examined after staining lightly with hematoxylin eosin azure. 


\section{Results}

Characterization of perfusate metabolites. When $\left[{ }^{3} \mathrm{H}\right] \mathrm{PAF}$ as a mixture of molecular species was instilled into the airway of the perfused lung, the radiolabel could be recovered in $98-100 \%$ yield of administered dose from perfusate and tissue extracts. During the time course of perfusion, a small amount of tritium was observed to rapidly appear in the nonrecirculating perfusate (see Fig. 1). This radiolabel component reached a maximum at $3.5 \mathrm{~min}$ after instillation. The eventual amount of radiolabel that appeared in the perfusate during the 15-min time course averaged only $4.1 \%$ of the instilled PAF.

Analysis of the perfusates pooled at three consecutive time points (0-5, 6-10, and 11-15 min) was carried out by the polar TLC system capable of separating lyso-PAF, PAF, and individual phospholipid classes. At all time points, the majority of radiolabel contained in the lung effluent was accounted for by PAF (Table I), and only a minor amount of lyso-PAF was observed. The proportion of $\mathrm{NL}$ in the perfusate was found to markedly increase during the 15 -min collection period to a level higher than that obtained in the first $5 \mathrm{~min}$. Because the PAF used here was prepurified to remove any traces of contaminating NL, this NL component actually represents a significant contribution to the metabolites appearing in the effluent.

The NL fraction in the lung effluent was further analyzed by a nonpolar TLC system capable of separating monoglycerides and diglycerides from the PAF diglyceride 1-0-alkyl-2-acetylglycerol. The monoglyceride fraction accounted for two-thirds of the radioactivity and was only 1-0-alkyl-glycerol, while the diglyceride fraction accounted for the remaining one-third, which was 1-0-alkyl-2-fatty acyl-glycerol. There was no metabolite corresponding to 1-0-alkyl-2-acetyl-glycerol.

Characterization of tissue-retained metabolites. Analysis of the lung tissue lipid extract on the polar TLC system is shown in Fig. 2, which revealed two major and three minor bands. The first minor band, representing $5 \%$ of the tissue radiolabel, had the same $\mathbf{R}_{\boldsymbol{f}}$ as authentic lyso-PAF. Structural identification of the radioactive components in this band was made by GC/MS as the isopropylidine derivative. Characteristic ions at 101 and $341 \mathrm{~m} / \mathrm{z}$ were found for a major component that had the correct gas chromatographic retention time for a 1-O-hexadecyl-monoglyceride isopropylidene derivative. The second minor band, band 4 , represented $\leq 2.5 \%$ of the tissue radioactivity and was found to co-migrate with glycerophosphoethanolamine on both TLC and normal-phase HPLC. It was not further characterized. A small amount of labeled NL was found within the tissue and analysis of this fraction by the nonpolar TLC system revealed a greater 1-0-alkyl-glycerol content than 1-0-alkyl-2-fatty acylglycerol.
Table I. Distribution of Perfusate Radioactivity* from $P A F^{\ddagger}$ Instilled in the Isolated Perfused Rat Lung during the Course of the 15-min Perfusion after Airway Instillation of PAF

\begin{tabular}{llll}
\hline & \multicolumn{2}{l}{$\%$ dpm in effluent } & \\
\cline { 2 - 4 } & $0-5 \mathrm{~min}$ & $6-10 \mathrm{~min}$ & $11-15 \mathrm{~min}$ \\
\hline & $\%$ & $\%$ & $\%$ \\
\% Total effluent & $(42.5 \pm 4.7)^{\| *}$ & $(35.4 \pm 1.5)$ & $(23.0 \pm 3.9)$ \\
Lyso-PAF & $11.7 \pm 2.3^{* *}$ & $11.0 \pm 0.9$ & $10.6 \pm 1.3$ \\
PAF & $73.3 \pm 3.1$ & $64.0 \pm 4.0$ & $52.8 \pm 2.2$ \\
NL & $12.9 \pm 1.4$ & $22.7 \pm 4.0$ & $32.8 \pm 3.9$
\end{tabular}

* $4 \%$ of the radiolabel from the total instilled PAF was recovered in the perfusate collected during the 15-min period.

${ }^{\ddagger}$ Radiolabeled PAF used was a mixture of molecular species.

${ }^{8}$ Lipid classes separated by the polar TLC system.

"Expressed as the percent of radioactivity appearing in the 5-min collection period as compared with the total perfusate radioactivity.

** Expressed as the percent of radioactivity in each lipid component during the specific time period.

'SEM, $n=5$.

One major component, band 2, corresponded to $10 \%$ of the tissue radiolabel and co-migrated with authentic PAF. Structural analysis of this radioactive component was made by negative ion electron capture GC/MS as the pentafluorobenzoate derivative. The characteristic molecular anion at $\mathrm{m} / \mathrm{z} 552$ was observed at the same retention time as that for trideuterio PAF, which was added to the lung in one experiment. This mass spectral data strongly support the presence of 1-O-hexadecyl-2-acetyl3-glycero-pentafluorobenzoate, which indicates that intact PAF occurred in this fraction.

The largest component, band 3, accounted for $81 \%$ of the retained label and was found as a broad fraction migrating with GPC containing long-chain acyl groups at the $s n-2$ position. Virtually identical results were obtained when $\left[{ }^{3} \mathrm{H}\right]$ lyso-PAF was instilled into the lung (Table II). The structures of the molecular species of the radiolabeled GPC were elucidated as described below.

Determination of ${ }^{3} \mathrm{H}$-labeled GPC molecular species. Fractionation of lung GPC as the diglyceride dinitrobenzoates by reverse-phase HPLC yielded a radiochromatogram containing two major and five minor peaks (from the metabolism of pure hexadecyl PAF, Fig. 3). The largest peak was found to have the same retention time as authentic 1- $O$-hexadecyl-2-arachidonoylGPC (16:Oe/20:4a-GPC). This single molecular species ac-

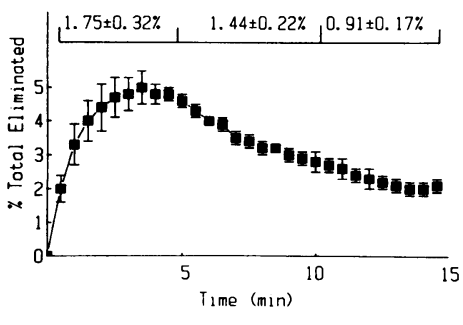

Figure 1. Appearance of radioactivity in rat lung perfusate after instillation of $\left[{ }^{3} \mathrm{H}\right] \mathrm{PAF}$ (100 ng) into the airway of the isolated perfused rat lung. The percent of injected radioactivity accumulated within 5-min intervals is indicated (errors indicated by SEM, $n=4$ ).
Figure 2. Thin-layer chromatographic separation of lung tissue-associated radioactivity (Bligh/Dyer extract). Radioactivity was scraped and pooled (bands 1-5) for further structural characterization as described in the text. PC, glycerophosphocholine; PE, glycerophosphoethanolamine. 
Table II. Distribution of Tissue-associated ${ }^{*}$ Radioactive Label from $P A F^{\ddagger}$ Instilled in the Isolated Perfused Rat Lung as Determined by TLC

\begin{tabular}{lcc}
\hline & \multicolumn{2}{l}{ Molecule instilled } \\
\cline { 2 - 3 } & PAF & Lyso-PAF" \\
\hline & $\%$ & $\%$ \\
Lyso-PAF* & $5.2 \pm 2.2^{1}(5)$ & 7.5 \\
PAF & $10.4 \pm 1.5(5)$ & ND** $^{* *}$ \\
GPC & $80.5 \pm 3.1(5)$ & 89.8 \\
GPE & $2.1 \pm 0.3(3)$ & 1.6 \\
NL & $2.2 \pm 0.3(4)$ & 1.1 \\
\hline
\end{tabular}

* $96 \%$ of radiolabel from the total instilled PAF was recovered in this fraction.

¿ Radiolabeled PAF used was a mixture of molecular species.

As described in text, each glycerophospholipid was further characterized by mass spectrometry for structural characterization. GPE, glycerophosphoethanolanine.

"Representative data from a single experiment.

'Standard error of the mean (number of experiments).

** Not detected.

counted for $24 \pm 1 \%$ of the total administered radiolabel. The second major peak in the radiochromatogram was found to coelute with that of authentic 1-O-hexadecyl-2-hexadecanoyl-GPC (16:Oe/16:Oa-GPC). This molecular species accounted for $24 \pm 3 \%$ of the labeled GPC fraction and $21 \pm 3 \%$ of the total administered radiolabel. Other minor species detected and their contribution to the radiolabeled GPC are listed in Table III. As can be seen in this table, the results for metabolism of $\left[{ }^{3} \mathrm{H}\right]$ lyso PAF were qualitatively similar to that of PAF. In experiments with pure hexadecyl PAF, these two metabolites corresponded

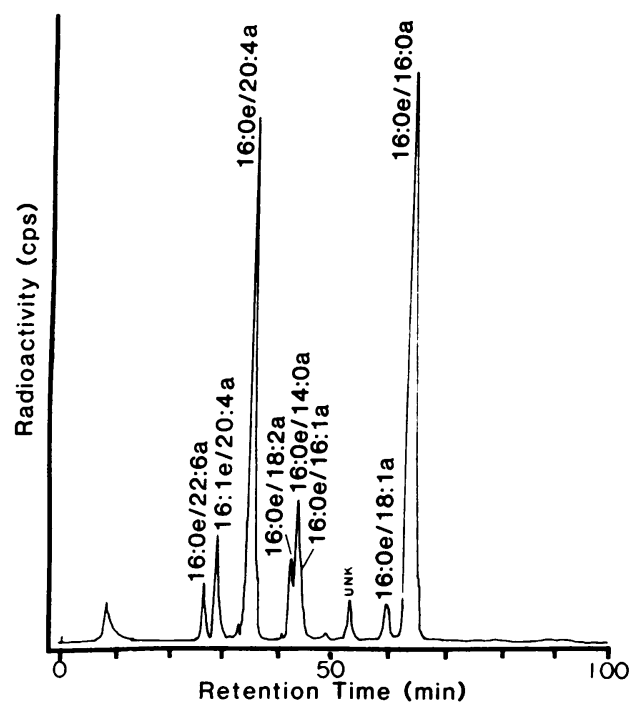

Figure 3. Reverse-phase HPLC separation of diglycerides (as dinitrobenzoate derivatives) from phospholipase $\mathrm{C}$ treatment of band 3 from the TLC shown in Fig. 2. Identification of GPC molecular species was based on HPLC retention time and negative ion electron capture mass spectrometry. Identification code: $\mathrm{C}_{\mathrm{mn-1}-1}: \mathrm{N}$ a or $\mathrm{e} / \mathrm{C}_{\mathrm{sn-2}}: \mathrm{N}$ a, where subscript $\mathbf{C}$ is the carbon chain number, $\mathbf{N}$ is degree of unsaturation, and a and e are acyl and alkyl ether, respectively. Unk, unknown.
Table III. Identification of GPC Molecular Species Metabolites of Radiolabeled PAF, ${ }^{*}$ Lyso-PAF, and DPPC Extracted from Lung Tissue after Instillation into the Airways of the Isolated Perfused Rat Lung ${ }^{\ddagger}$

\begin{tabular}{lcccc}
\hline \multicolumn{5}{c}{ Material instilled } \\
\cline { 2 - 5 } Molecular species & $\begin{array}{l}\text { PAF } \\
\text { (hexadecyl)* }\end{array}$ & PAF (mixture) & Lyso PAF" & DPPC \\
\hline & $\%$ & $\%$ & $\%$ & $\%$ \\
16:Oe/22:6a & 3.0 & $5.8 \pm 0.1$ & 7.3 & ND' \\
16:1e/20:4 & 5.5 & $6.3 \pm 0.5$ & 7.3 & ND \\
16:Oe/20:4a & 27 & $31.4 \pm 2.4$ & 37.8 & ND \\
18:Oe/18:3a & - & $3.9 \pm 0.3$ & - & ND \\
16:Oe/18:2a; & 15 & $12.0 \pm 0.3$ & 11 & ND \\
16:Oe/16:1a $+14: O a^{* *}$ & & & & \\
18:Oe/20:4a & - & $2.9 \pm 0.4$ & 3.2 & ND \\
16:Oe/18:1a & 3.4 & $2.4 \pm 0.5$ & 0.4 & ND \\
16:Oe/16:Oa & 39 & $23.7 \pm 0.7$ & 21.4 & ND \\
Lyso & $5 \neq$ & $4.1 \pm 0.8$ & 4.2 & ND \\
16:Oa/16:Oa & - & - & - & 97 \\
Others (unidentified) & 2.5 & 8.4 & 7.5 & 3.0 \\
& & & & \\
\hline
\end{tabular}

* The single molecular species 1-0-hexadecyl-2-acetyl-sn-glycerophosphocholine of PAF was instilled.

${ }^{\ddagger}$ Relative amount of radioactivity for each metabolite is expressed as percent of total radioactivity in the GPC fraction. Identifications of each molecular species were carried out by negative ion electron capture mass spectrometry of the dinitro-benzoates separated by HPLC. ${ }^{8}$ A mixture of molecular species of PAF described in Methods was instilled. SEM, $n=4$, not resolved; given as summation peaks.

"A mixture of molecular species of lyso-PAF was instilled.

1 Not detected.

** Not fully resolved; given as summation of components.

${ }^{\ddagger}$ HPLC retention time for lysophosphatidylcholine, but not further characterized.

to $27 \%(16: \mathrm{Oe} / 20: 4 \mathrm{a}-\mathrm{GPC})$ and $39 \%(16: \mathrm{Oe} / 16: \mathrm{Oa}-\mathrm{GPC})$ of the labeled GPC fraction.

To unequivocally assign molecular structures to these molecules, the dinitrobenzoate derivatives were analyzed by negative ion desorption chemical ionization as well as HPLC retention times. Since an abundant molecular anion $\left(\mathrm{M}^{-}\right)$is produced by this process, the radyl group at $s n-2$ of the diglyceride can be readily assigned. The ion at $798 \mathrm{~m} / \mathrm{z}$ (Fig. 4, top) corresponded to 1-O-hexadecyl-2-arachidonoyl-3-dinitrobenzoate, and at 784 $\mathrm{m} / \mathrm{z}$ (Fig. 4, bottom) corresponded to 1- $O$-hexadecyl-2-hexadecanoyl-3-dinitrobenzoate. Molecular species assignment for the minor radiolabeled GPC metabolites were deduced in this manner.

To determine if the metabolism of PAF into alkyl, acyl-GPC was specific, a radiolabeled diacyl species, DPPC, was administered in an identical manner to that for PAF. In this instance, DPPC was found to undergo no metabolism at all (Table III) and was detected as intact DPPC from lung lipid by RP-HPLC and fast atom bombardment mass spectrometry (data not shown) using the method of Chilton and Murphy (21).

Autoradiographic identification of cells containing PAF metabolites. To suggest the major sites of PAF metabolism in the lung and to determine whether there were cells that preferentially metabolized $\left[{ }^{3} \mathrm{H}\right] \mathrm{PAF}$, lungs were subjected to light level auto- 

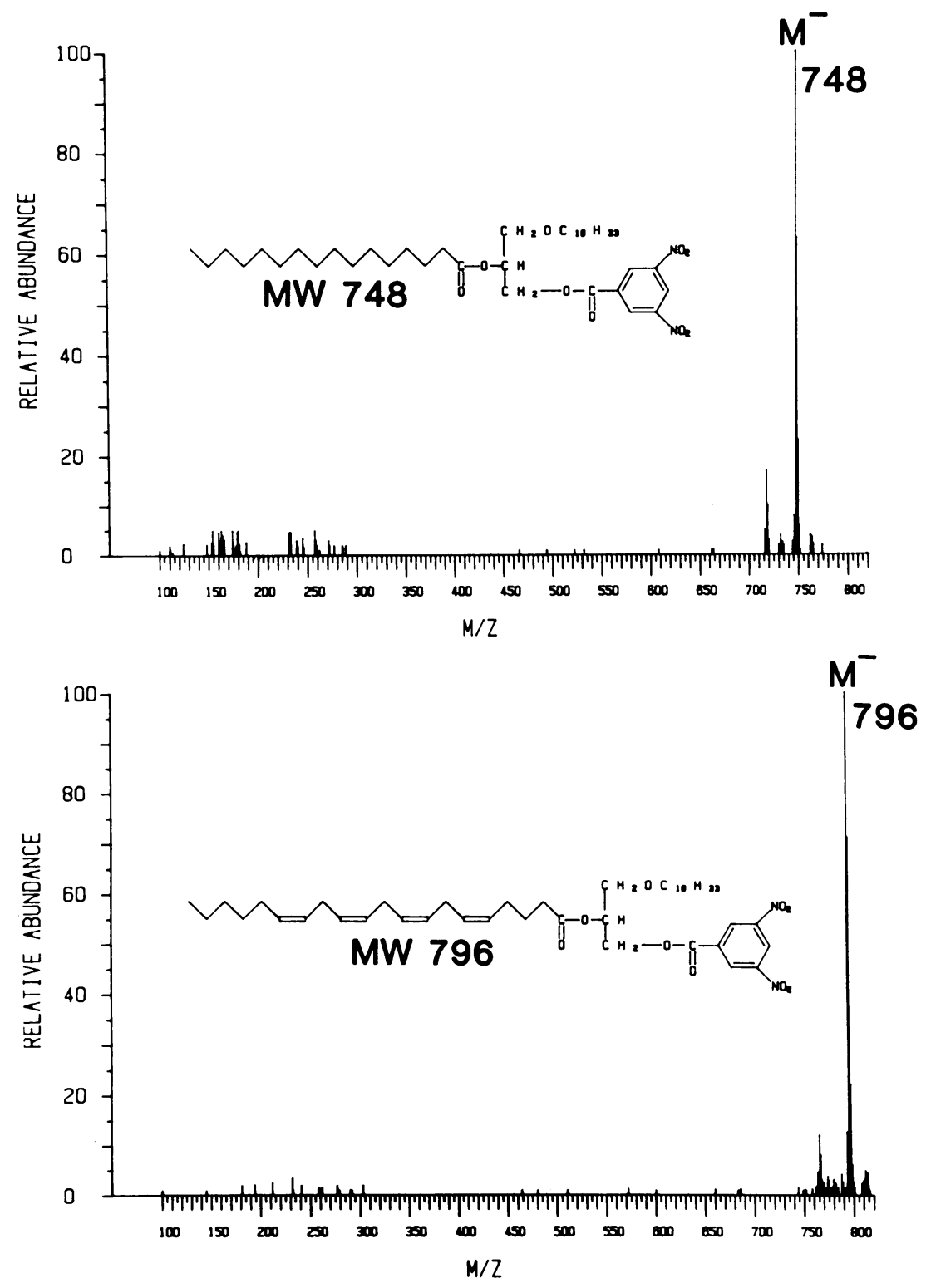

Figure 4. Negative ion electron capture mass spectra obtained from radioactive PAF metabolites eluting at (top) $26.5 \mathrm{~min}$ and (bottom) $14.0 \mathrm{~min}$ from the reverse-phase HPLC separation. The molecular anions $\left(\mathrm{M}^{-}\right)$observed correspond to the structures indicated for each mass spectrum. radiography after the perfusion protocol at the exact same time period that had been used for the biochemical determinations. In the conducting portion of the airways, the majority of radiolabel was found (Fig. $5 A$ ) to be sequestered preferentially within the nonciliated bronchiolar epithelial cells (Clara cells) (27). Clearly, the nonciliated epithelial cells highly concentrated the radiolabeled lipid, whereas the ciliated epithelial cells were essentially devoid of label. Within the alveolar air spaces (Fig. 5 $B$ ), the radiolabel was observed to be highly concentrated to one particular cell type, apparently the type II epithelial cell. Minimal label was found to be associated with the alveolar macrophage (Fig. $5 C$ ), type I epithelial cells, endothelial cells, or interstitial cells. To establish whether this phenomenon was specific for PAF or a property of GPC in general, the distribution of radiolabeled DPPC by autoradiography was studied for comparison. In contrast to PAF instillation, no cell type within the conducting airways or alveolar air space was seen to uniquely concentrate DPPC (Fig. $5 D$ ). The radiolabeled DPPC was found to be evenly distributed in essentially all cell types.

\section{Discussion}

The present study demonstrates that PAF is rapidly and extensively metabolized by the isolated perfused lung. In these experiments, recovery of radiolabeled PAF was close to quantitative; $96 \%$ of the instilled PAF was retained by the lung, $81 \%$ of which was found to be converted to long-chain acyl GPC species. The remaining $4 \%$ of the radioactive tracer traversed the lung cellular barriers to appear in the perfusate. This rapid conversion of PAF into alkylacyl-GPC species retained in tissues has so far been described only in isolated cells (13-18). In one previous study involving the metabolism of intravenously injected PAF (12), the lung was found to accumulate the highest amount of injected radioactivity compared with all other major organs. In that study the radiolabel within the lung was associated with alkyl, acyl-GPC. Hence, it appears that the metabolic profile of PAF at the whole animal and organ level is qualitatively similar to that described in isolated cells.

The finding that the human neutrophil $(13,14)$ and alveolar 

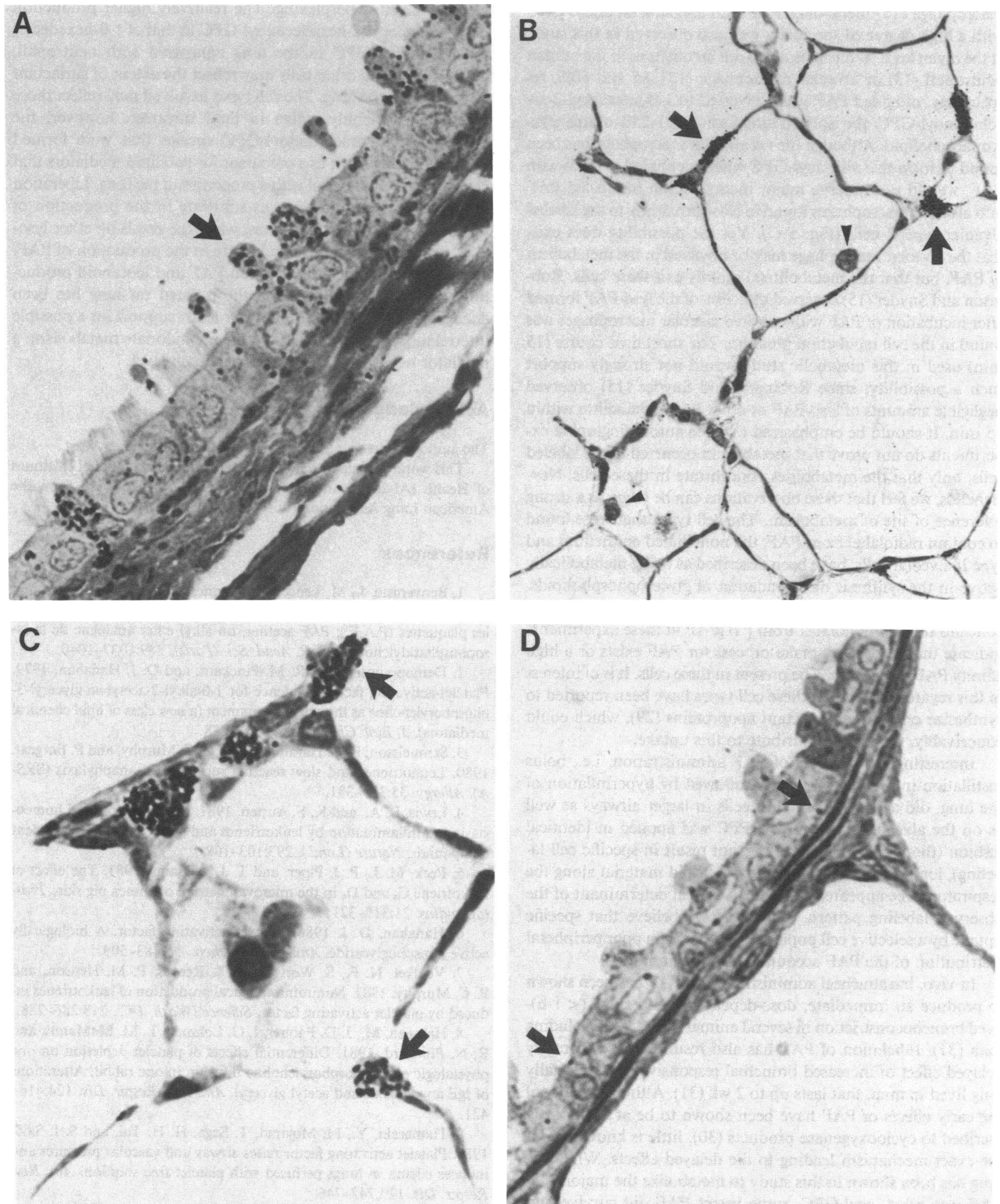

Figure 5. Microscope autoradiograph of tissue slices from the rat lung instilled with $\left[{ }^{3} \mathrm{H}\right] \mathrm{PAF} 15 \mathrm{~min}$ earlier. (A) Radiolabel (grains) appearing in the nonciliated bronchiolar epithelial cell (Clara cell). (B) Radioactivity appearing in a presumed type II cell in the alveolar wall. (C) Magnification of the alveolar region indicating radioactivity

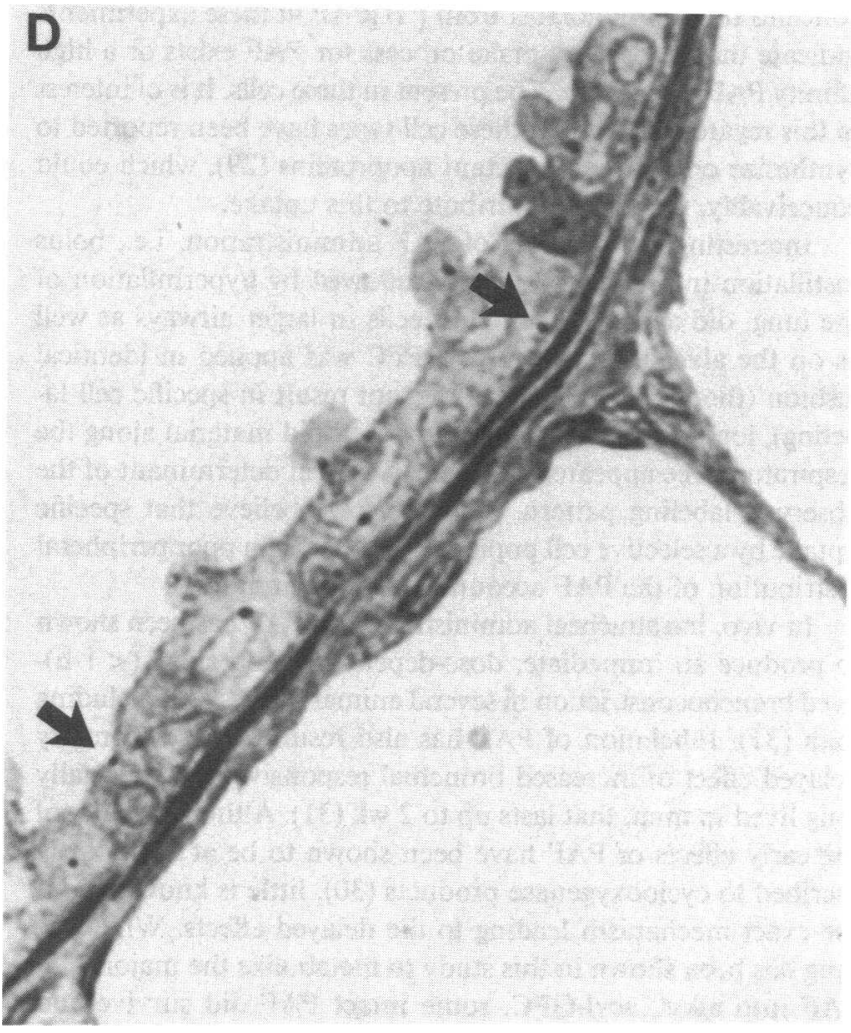

(grains) from PAF appearing in the presumed type II cell but not in the alveolar macrophage in the alveolar space. $(D)$ Radiolabel (grains) from $\left[{ }^{3} \mathrm{H}\right] \mathrm{DPPC}$ instilled in an identical manner to PAF. The autoradiograph was taken at the airway level similar to that shown in $A$. 
macrophage (15) metabolize PAF into alkylarachidonoyl-GPC with a high degree of specificity was also observed in this study at the organ level. While in isolated cell incubations of the human neutrophil (13) or alveolar macrophage (15), 85 and 60\%, respectively, of added PAF was converted to 1-0-hexadecyl-2-arachidonoyl-GPC; the isolated lung formed $20-25 \%$ of this glycerophospholipid. Although the rat alveolar macrophage has been found to form this alkylacyl-GPC when incubated in vitro with PAF, we did not observe major incorporation of labeled PAF into alveolar macrophages found in close proximity to the labeled alveolar type II cells (Fig. $5 \mathrm{C}$ ). Yet the possibility does exist that the alveolar macrophage may be involved in the metabolism of PAF, but that the metabolite(s) rapidly exit these cells. Robinson and Snyder (15) observed that $90 \%$ of the lyso-PAF formed after incubation of PAF with cultured alveolar macrophages was found in the cell incubation medium. The short time course (15 min) used in this metabolic study would not strongly support such a possibility, since Robinson and Snyder (15) observed negligible amounts of lyso-PAF or other PAF metabolites within $15 \mathrm{~min}$. It should be emphasized that the autoradiographic experiments do not prove that metabolism occurred in the labeled cells, only that the metabolites concentrate in these cells. Nevertheless, we feel that these observations can be taken as a strong inference of site of metabolism. The cell types that were found to contain radiolabel from PAF, the nonciliated epithelium and type II alveolar cells, have been described as being metabolically active in the synthesis or degradation of glycerophospholipids, in particular, lung surfactant (28). It is likely that the unique concentration of radiolabel from $\left[{ }^{3} \mathrm{H}\right] \mathrm{PAF}$ in these experiments indicate that a specific uptake process for PAF exists or a high affinity PAF receptor may be present in these cells. It is of interest in this regard that both of these cell types have been reported to synthesize one of the surfactant apoproteins (29), which could conceivably, therefore, contribute to this uptake.

Interestingly, the mode of PAF administration, i.e., bolus instillation into a central airway followed by hyperinflation of the lung, did achieve labeling of cells in larger airways as well as on the alveolar level. Since DPPC was applied in identical fashion (though its deposition did not result in specific cell labeling), longitudinal distribution of the lipid material along the respiratory tree appeared not to be a critical determinant of the observed labeling pattern. Therefore, we believe that specific uptake by a selective cell population rather than poor peripheral distribution of the PAF accounted for our findings.

In vivo, intratracheal administration of PAF has been shown to produce an immediate, dose-dependent, and short $(<1 \mathrm{~h})$ lived bronchoconstriction in several animal species (30) including man (31). Inhalation of PAF has also resulted in a secondary delayed effect of increased bronchial responsiveness, especially long lived in man, that lasts up to 2 wk (31). Although many of the early effects of PAF have been shown to be at least partly ascribed to cyclooxygenase products (30), little is known about the exact mechanism leading to the delayed effects. While the lung has been shown in this study to metabolize the majority of PAF into alkyl, acyl-GPC, some intact PAF did survive and remain within certain cells.

The rapid metabolism of PAF into long-chain acyl GPC molecular species may reflect an inactivation mechanism to protect the lung from this agent, which has been shown to have marked pharmacologic effects on this organ (31). However, the reason for the observed specificity of reacylation and the cell types involved is perplexing. The relatively higher production of 1-0-hexadecyl-2-hexadecanoyl-GPC to that of 1-0-hexadecyl2-arachidonoyl-GPC in the lung compared with neutrophil, macrophage, and other cells may reflect the extent of surfactant production in the lung. The cell types involved may reflect those cells that are highly active in lipid turnover; however, the 1-0-hexadecyl-2-arachidonoyl-GPC species that were formed may potentially serve as a precursor for two lipid mediators that have profound biological active properties in the lung. Liberation of arachidonate could serve as substrate in the production of icosanoids, such as leukotrienes, while the resulting ether lysoGPC could further serve as substrate in the production of PAF. The potential connection between PAF and icosanoid production in isolated cells (13) and the isolated rat lung has been documented (7). This study further lends support for a possible interrelationship between PAF and arachidonate metabolism, a mediator network (32) at the whole organ level.

\section{Acknowledgments}

The authors appreciate the technical assitance of Stephen West.

This work was supported in part by grants from National Institutes of Health (AI-20774 and HL-34303) and a Career Award from the American Lung Association (to N. F. Voelkel).

\section{References}

1. Benveniste, J., M. Tence, P. Varenne, J. Bidault, C. Boullet, and J. Polonsky. 1979. Semi-synthese et stracture proposee du facteur activant les plaquettes (P.A.F.): PAF-acether, un alkyl ether analogue de la lysophosphatidylcholine. C. R. Acad. Sci. (Paris). 289:1037-1040.

2. Demopoulos, C. A., R. M. Pinckard, and D. J. Hanahan. 1979. Platelet-activating factor. Evidence for 1-0-alkyl-2-acetyl-sn-glyceryl-3phosphorylcholine as the active component (a new class of lipid chemical mediators). J. Biol. Chem. 254:9355-9358.

3. Samuelsson, B., S. Hammarstrom, R. C. Murphy, and P. Borgeat. 1980. Leukotrienes and slow reacting substance of anaphylaxis (SRSA). Allergy. 35:375-381.

4. Lewis, R. A., and K. F. Austen. 1981. Mediation of local homeostasis and inflammation by leukotrienes and other mast cell-dependent compounds. Nature (Lond.). 293:103-108.

5. Peck, M. J., P. J. Piper, and T. J. Williams. 1981. The effect of leukotriene $\mathrm{C}_{4}$ and $\mathrm{D}_{4}$ in the microvasculature of guinea pig skin. Prostaglandins. 21:315-321.

6. Hanahan, D. J. 1986. Platelet activating factor: A biologically active phosphoglyceride. Ann. Rev. Biochem. 55:483-509.

7. Voelkel, N. F., S. Worthen, J. T. Reeves, P. M. Henson, and R. C. Murphy. 1982. Nonimmunological production of leukotrienes induced by platelet activating factor. Science (Wash. DC). 218:286-288.

8. Halonen, M., J. D. Palmer, I. G. Lohman, L. M. McManus, and R. N. Pinckard. 1981. Differential effects of platelet depletion on one physiologic ether phosphorylcholine infusion in one rabbit. Alterations of IgE anaphylaxis and acetyl glyceryl. Am. Rev. Respir. Dis. 124:416421.

9. Hamasaki, Y., M. Mojarad, T. Saga, H. H. Tai, and S. I. Said. 1984. Platelet activating factor raises airway and vascular pressures and induces edema in lungs perfused with platelet free solution. Am. Rev. Respir. Dis. 129:742-746.

10. Voelkel, N. F., S. Chang, K. Pfeffer, S. G. Worthen, I. F. McMurty, and P. M. Henson. 1986. PAF antagonists: Different effects on platelets, neutrophils, guinea pig ileum, and PAF-induced vasodilation in isolated rat lung. Prostaglandins. 32:359-373.

11. Harper, T. W., J. Y. Wescott, N. F. Voelkel, and R. C. Murphy. 1984. Metabolism of leukotrienes $B_{4}$ and $C_{4}$ in the isolated perfused rat lung. J. Biol. Chem. 259:14437-14440. 
12. Blank, M. L., E. A. Cress, T. Whittle, and F. Snyder. 1981. In vivo metabolism of a new class of biologically active phospholipids: 1alkyl-2-acetyl-sn-glycero-3-phosphocholine, A platelet activating-hypotensive phospholipid. Life Sci. 29:769-775.

13. Chilton, F. H., J. T. O'Flaherty, J. M. Ellis, C. L. Swendsen, and R. L. Wykle. 1982. Metabolic fate of platelet-activating factor in neutrophils. J. Biol. Chem. 258:6357-6361.

14. Chilton, F. H., J. T. O'Flaherty, J. M. Ellis, C. L. Swendsen, and R. L. Wykle. 1983. Selective acylation of lyso platelet activating factor by arachidonate in human neutrophils. J. Biol. Chem. 258:7268-7271.

15. Robinson, M., and F. Snyder. 1985. Metabolism of platelet-activating factor by rat alveolar macrophages: Lyso-PAF as an obligatory intermediate in the formation of alkylarachidonoylglycerophosphocholine species. Biochim. Biophys. Acta. 837:52-56.

16. Blank, M. L., A. A. Spector, T. L. Kaduce, T.-C. Lee, and F. Snyder. 1986. Metabolism of platelet activating factor (1-alkyl-2-acetylsn-glycero-3-phosphocholine) and 1-alkyl-2-acetyl-sn-glycerol by human endothelial cells. Biochim. Biophys. Acta. 876:373-378.

17. Tan, E. L., and F. Snyder. 1985. Metabolism of platelet activating factor (1-alkyl-2-acetyl-sn-glycero-3-phosphocholine) by capillary endothelial cells isolated from rat epididymal adipose tissue. Thromb. Res. 38:713-717.

18. Kramer, R. M., G. M. Patton, C. R. Pritzker, and D. Deykin. 1984. Metabolism of platelet activating factor in human platelets. $J$. Biol. Chem. 259:13316-13320.

19. Hoffman, D. R., C. T. Truong, and J. M. Johnston. 1986. The role of platelet-activating factor in human fetal lung maturation. Am. J. Obstet. Gynecol. 155:70-75.

20. Clay, K. L., R. C. Murphy, J. L. Andres, J. Lynch, and P. M. Henson. 1984. Structure elucidation of platelet activating factor derived from human neutrophils. Biochem. Biophys. Res. Commun. 121:815825.

21. Chilton, F. H., and R. C. Murphy. 1986. Fast atom bombardment analysis of arachidonic acid-containing phosphatidylcholine molecular species. Biomed. Environ. Mass Spec. 13:71-76.

22. Voelkel, N. F., J. G. Gerber, I. F. McMurtry, A. S. Nies, and J. T. Reeves. 1981. Release of vasodilator prostaglandin, $\mathrm{PGI}_{2}$, from isolated rat lung during vasoconstriction. Circ. Res. 48:207-213.

23. Bligh, E. G., and W. J. Dyer. 1959. A rapid method of total lipid extraction and purification. Can. J. Biochem. Physiol. 37:911-917.

24. Haroldsen, P. E., K. L. Clay, and R. C. Murphy. 1987. Quantitation of lyso-platelet activating factor molecular species from human neutrophils by mass spectrometry. J. Lipid Res. 28:42-49.

25. Ramesha, C. S., and W. C. Pickett. 1986. Measurement of subpicogram quantities of platelet activating factor (AGEPC) by gas chromatography/negative ion chemical ionization mass spectrometry. Biomed. Environ. Mass Spectrom. 13:107-111.

26. Kito, M. H., H. Takamura, H. Narita, and R. Urade. 1985. A sensitive method for quantitative analysis of phospholipid molecular species by high-performance liquid chromatography. J. Biochem. 98: 327-331.

27. Plopper, C. G. 1983. Comparative morphologic features of bronchiolar epithelial cells. Am. Rev. Respir. Dis. 128:537-541.

28. Goerke, J. 1974. Lung surfactant. Biochim. Biophys. Acta. 344: 241-261.

29. King, R. J. 1984. The apoproteins of pulmonary surfactant. Prog. Respir. Res. 18:68-82.

30. Chung, K. F., H. Aizawa, G. D. Leikauf, I. F. Veki, T. W. Evans, and J. A. Nadel. 1986. Airway hyperesponsiveness induced by platelet activating factor: Role of thromboxane generation. J. Pharmacol. Exp. Ther. 236:580-584.

31. Cuss, F. M., C. M. S. Dixon, and P. J. Barnes. 1986. Effects of inhaled platelet activating factor on pulmonary function and bronchial responsiveness in man. Lancet. ii:189-192.

32. Murphy, R. C., and P. M. Henson. 1985. Mediator network. Ann. Inst. Pasteur Immunol. 136:219-221. 